\title{
The spectra of exotic light mass nuclei determined with MCAS theory
}

\author{
K. Amos ${ }^{1, \mathrm{a}}$, L. Canton ${ }^{2}$, G. Pisent ${ }^{2}$, J.P. Svenne ${ }^{3}$, and S. Karataglidis ${ }^{4}$ \\ 1 School of Physics, University of Melbourne, Victoria 3010, Australia \\ 2 Istituto Nazionale di Fisica Nucleare, Sezione di Padova, e Dipartimento di Fisica dell’Università di Padova, \\ via Marzolo 8, 35131 Padova, Italy \\ 3 Department of Physics and Astronomy, University of Manitoba, and Winnipeg Institute for Theoretical Physics, \\ Winnipeg, Manitoba, Canada R3T 2N2 \\ 4 Department of Physics and Electronics, Rhodes University, Grahamstown 6140, South Africa
}

\begin{abstract}
A Multi-Channel Algebraic Scattering (MCAS) theory has been used to study the interaction of a nucleon with light-mass nuclei as a coupled-channel problem. The method can be solved for all energies so that bound states as well as resonances of the compound nucleus can be specified, even if the compound nucleus is nucleon unstable. A resonance finding prescription ensures that all resonances of the system can be found no matter how weak and/or narrow. Spectra of mass-7 nuclei and of the proton unstable ${ }^{15} \mathrm{~F}$ are discussed, the Coulomb displacement energy considered, and first results on using MCAS details in a radiative capture cross section calculation presented.
\end{abstract}

\section{Introduction}

The MCAS method [1,2] solves coupled channel LippmannSchwinger equations for a chosen two-body system. The theory is built upon sturmian expansions of whatever one chooses to be the interaction matrix of potential functions for the system. The set of sturmians that form the basis of the expansions are determined from the self-same interaction of potentials and, if necessary, can be selected to ensure that the Pauli principle is not violated. The approach, most suited to deal with low energies, is noteworthy because its formulation facilitates a systematic determination of all sub-threshold bound states and compound resonances within any energy range considered.

While the required starting matrix of potentials may be constructed from any model of nuclear structure, to date we have used just a simple collective rotational to define those potentials with deformation taken to second order. Also, to date the ground, first, and second excited states of the target nucleus have been used to form the channel couplings.

Isospin symmetry in mirror scattering systems, save only for inclusion/exclusion of the Coulomb interaction, has been used with MCAS to estimate the spectra of nuclei that are just outside of the proton drip line. ${ }^{15} \mathrm{~F}$ is one such case [3]. The $\mathrm{A}=7$ system also has been considered with a unique nuclear potential, and the level structures of ${ }^{7} \mathrm{He},{ }^{7} \mathrm{Li},{ }^{7} \mathrm{Be}$, and ${ }^{7} \mathrm{~B}$ have been obtained [2]. We have also used the scheme to consider low energy properties of ${ }^{3} \mathrm{H}$ - and ${ }^{3} \mathrm{He}-{ }^{4} \mathrm{He}$ scattering to correlate different views of the spectra of ${ }^{7} \mathrm{Li}$ and ${ }^{7} \mathrm{Be}$. The di-cluster structure of ${ }^{7} \mathrm{Be}$ that results has then been used to evaluate the astrophysical $\mathrm{S}$-factor for the radiative capture of ${ }^{3} \mathrm{He}$ by ${ }^{4} \mathrm{He}$.

The Coulomb field has been taken simply as that of a uniform charged sphere but we have now allowed deformation of the Coulomb field (again to second order) in defining the initiating potentials. From the results of MCAS evaluations,

\footnotetext{
${ }^{a}$ Presenting author, e-mail: amos@physics . unimelb.edu.au
}

Coulomb displacement energies for the mass-13 mirror nuclei have been extracted.

\section{The MCAS theory}

In brief, the MCAS approach is based upon using sturmian functions (Weinberg states) as a basis set to expand the chosen interaction potentials. Each interaction matrix then has the form of a sum of separable interactions. The analytic properties of the $S$ matrix from a separable Schrödinger potential gives the means by which a full algebraic solution of the multichannel scattering problem can be realized. All details of the MCAS theory have been published [1] and so only salient features are repeated herein. Consider a coupledchannel system for each allowed scattering spin-parity $J^{\pi}$. With the MCAS method, one solves the Lippmann-Schwinger (LS) integral equations in momentum space, i.e.,

$$
\begin{aligned}
& T_{c c^{\prime}}(p, q ; E)=V_{c c^{\prime}}(p, q)+ \\
& \frac{2 \mu}{\hbar^{2}}\left[\sum_{c^{\prime \prime}=1}^{\text {open }} \int_{0}^{\infty} V_{c c^{\prime \prime}}(p, x) \frac{x^{2}}{k_{c^{\prime \prime}}^{2}-x^{2}+i \epsilon} T_{c^{\prime \prime} c^{\prime}}(x, q ; E) d x\right. \\
& \left.-\sum_{c^{\prime \prime}=1}^{\text {closed }} \int_{0}^{\infty} V_{c c^{\prime \prime}}(p, x) \frac{x^{2}}{h_{c^{\prime \prime}}^{2}+x^{2}} T_{c^{\prime \prime} c^{\prime}}(x, q ; E) d x\right],
\end{aligned}
$$

where the index $c$ denotes the quantum numbers that identify each channel uniquely. Such requires specification of potential matrices $V_{c c^{\prime}}^{\left(J^{\pi}\right)}(p, q)$. The open and closed channels have channel wave numbers $k_{c}$ and $h_{c}$ for $E>\epsilon_{c}$ and $E<\epsilon_{c}$ respectively. $\mu$ is the reduced mass. Solutions of equation (1) are sought using expansions of the potential matrix elements in (finite) sums of energy-independent separable terms,

$$
V_{c c^{\prime}}(p, q) \sim \sum_{n=1}^{N} \chi_{c n}(p) \eta_{n}^{-1} \chi_{c^{\prime} n}(q) .
$$


The key to the method is the choice of the expansion form factors $\chi_{c n}(q)$. Optimal ones have been found from the sturmian functions that are determined from the actual (coordinate space) model interaction $V_{c c^{\prime}}(r)$ initially chosen to describe the coupled-channel problem.

The link between the multichannel $T$ - and the scattering $(S$-) matrices involves a Green's function matrix,

$$
\begin{aligned}
\left(G_{0}\right)_{n n^{\prime}}=\frac{2 \mu}{\hbar^{2}}[ & \sum_{c=1}^{\text {open }} \int_{0}^{\infty} \chi_{c n}(x) \frac{x^{2}}{k_{c}^{2}-x^{2}+i \epsilon} \chi_{c n^{\prime}}(x) d x \\
& \left.-\sum_{c=1}^{\text {closed }} \int_{0}^{\infty} \chi_{c n}(x) \frac{x^{2}}{h_{c}^{2}+x^{2}} \chi_{c n^{\prime}}(x) d x\right],
\end{aligned}
$$

with a diagonal eigenvalue matrix, $(\eta)_{n n^{\prime}}=\eta_{n} \delta_{n n^{\prime}}$. The bound states of the compound system are defined by the zeros of the matrix determinant for energy $E<0$. They link to the zeros of $\left\{\left|\boldsymbol{\eta}-\mathbf{G}_{0}\right|\right\}$ when all channels in equation (3) are closed.

Elastic scattering observables follow from the on-shell properties $\left(k_{1}=k_{1}^{\prime}=k\right)$ of the scattering matrices. For the elastic scattering of neutrons (spin $\frac{1}{2}$ ) from spin zero targets $c=c^{\prime}=1$, and $S_{11} \equiv S_{\ell}^{J}=S_{\ell}^{( \pm)}$are

$$
\begin{aligned}
S_{11}=1-i \pi & \frac{2 \mu k}{\hbar^{2}} \sum_{n n^{\prime}=1}^{M} \chi_{1 n}(k) \\
& \times \frac{1}{\sqrt{\eta_{n}}}\left[\left(\mathbf{1}-\boldsymbol{\eta}^{-\frac{1}{2}} \mathbf{G}_{0} \boldsymbol{\eta}^{-\frac{1}{2}}\right)^{-1}\right]_{n n^{\prime}} \frac{1}{\sqrt{\eta_{n^{\prime}}}} \chi_{1 n^{\prime}}(k) .
\end{aligned}
$$

Diagonalization of the complex-symmetric matrix,

$$
\sum_{n^{\prime}=1}^{N} \eta_{n}{ }^{-\frac{1}{2}}\left[\mathbf{G}_{0}\right]_{n n^{\prime}} \eta_{n^{\prime}}{ }^{-\frac{1}{2}} \tilde{Q}_{n^{\prime} r}=\zeta_{r} \tilde{Q}_{n r}
$$

establishes the evolution of the complex eigenvalues $\zeta_{r}$ with respect to energy. Resonant behavior occurs when an eigenvalue $\zeta_{r}$ crosses the unit circle in the Gauss plane near the point $(1,0)$.

\section{Results}

\subsection{The spectrum of ${ }^{15} \mathrm{~F}\left(p+{ }^{14} \mathrm{O}\right)$}

Spectra, known and calculated using MCAS [3], for mass 15 are shown in figure 1 . The known properties of ${ }^{15} \mathrm{C}$ agree well with our results for the $n+{ }^{14} \mathrm{C}$ system. See ref. [3] for details. The spectrum of ${ }^{15} \mathrm{C}$ has two bound states of spinparities $\frac{1}{2}^{+}$(ground) and $\frac{5}{2}^{+}$. They are described dominantly as a single $s d$ shell neutron on the ${ }^{14} \mathrm{C}$ ground state. Then there are three quite narrow resonances, all having negative parity, which lie within the spread of a broad $\frac{3}{2}^{+}$resonant state. That broad $\frac{3}{2}^{+}$was seen very clearly in the cross section from a measurement of the ${ }^{14} \mathrm{C}(d, p)$ reaction. It is noteworthy that there are no other bound states; in particular none having negative parity. Such would occur if, in the $\mathrm{n}+{ }^{14} \mathrm{C}$ system, the $0 p_{\frac{1}{2}}$ neutron orbit were not Pauli-blocked. However, there are negative parity resonances and to find them in our evaluations

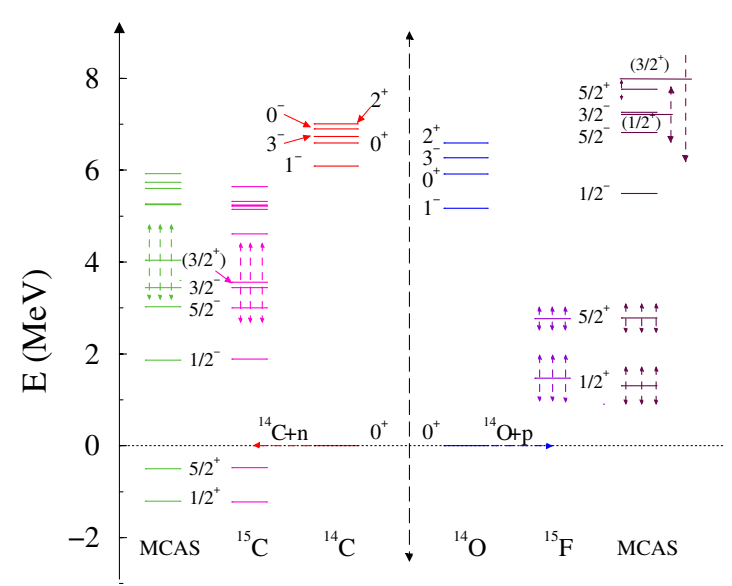

Fig. 1. Low energy spectra of ${ }^{14,15} \mathrm{C}$ and of ${ }^{14,15} \mathrm{O}$, and from results of our MCAS calculations. The zero of energy is set to that of the relevant mass-14 ground states.

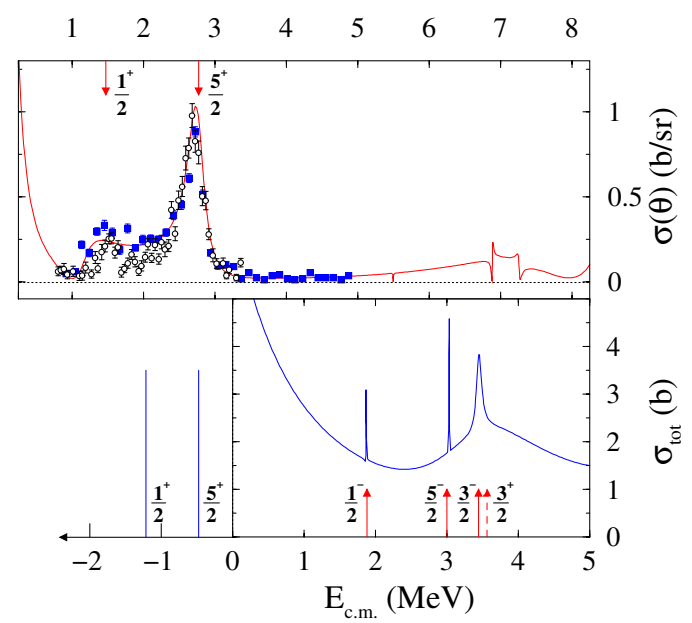

Fig. 2. The elastic cross sections from scattering of ${ }^{14} \mathrm{O}$ ions from hydrogen at $\theta_{c . m .}=180^{\circ}$ (top) and our predicted total cross section for $n-{ }^{14} \mathrm{C}$ scattering (bottom). Arrows indicate known states.

of ${ }^{15} \mathrm{C}$ required that the neutron $0 p_{\frac{1}{2}}$ orbit only be Pauli hindered [3]. With the nuclear interaction set, the results from MCAS evaluations of the $p+{ }^{14} \mathrm{O}$ system to form the particle unstable ${ }^{15} \mathrm{~F}$ are shown on the right of this figure.

The two lowest resonance states of ${ }^{15} \mathrm{~F}$ have been observed recently in scattering cross-sections. Those data and our results are shown in figure 2 . In the top panel we show the cross sections from ${ }^{14} \mathrm{O}$ scattering from hydrogen. Our MCAS result (solid curve) compares very well with the recent data and is as good as has been found with other analyses [3]. In the bottom panel of figure 2 is our prediction of the total scattering cross section of neutrons from ${ }^{14} \mathrm{C}$ for energies to $5 \mathrm{MeV}$. There are four obvious resonances; three quite narrow (of negative parity) and a very broad $\frac{3}{2}^{+}$one. The three narrow resonances have partners in the $p+{ }^{14} \mathrm{O}$ cross section that is shown in the top panel, while the broad $\frac{3}{2}^{+}$structure becomes more complex and overlaps with two other states. The zero of the energy scale has been placed to optimally match the $\frac{5}{2}^{+}$ bound state in ${ }^{14} \mathrm{C}$ to the centroid of the analogous resonance state in ${ }^{15} \mathrm{~F}$. 
Analogues of the negative parity resonances in ${ }^{15} \mathrm{C}$ are predicted in ${ }^{15} \mathrm{~F}$. But the origin of these new, narrow negative parity resonances in ${ }^{15} \mathrm{~F}$ differs from those of the observed low-lying ones. They are compound resonances and they are due to the Pauli-hindrance of proton $0 p_{\frac{1}{2}}$ orbit in the $0_{2}^{+}$and $2^{+}$excited states of ${ }^{14} \mathrm{O}$.

\subsection{Spectra of ${ }^{7} \mathrm{He},{ }^{7} \mathrm{Li},{ }^{7} \mathrm{Be},{ }^{7} \mathrm{~B}$}

\subsubsection{Spectra from nucleon-mass 6 coupling}

A single nuclear interaction matrix of potentials was found whose use with appropriate Coulomb terms in MCAS calculations give spectra of these nuclei in good agreement with known ones. For ${ }^{7} \mathrm{Li}\left(\mathrm{p}+{ }^{6} \mathrm{He}\right)$ and ${ }^{7} \mathrm{Be}\left(\mathrm{n}+{ }^{6} \mathrm{Be}\right)$ the results are listed in table 1 . Therein the numbers in square brackets

Table 1. Bound spectra of ${ }^{7} \mathrm{Li}$ and of ${ }^{7} \mathrm{Be}$.

\begin{tabular}{ccccc}
\hline$J^{\pi}$ & ${ }^{7}$ Li Exp. & Theory & ${ }^{7}$ Be Exp. & Theory \\
\hline$\frac{3}{2}^{-}$ & -10.0 & -10.0 & -10.7 & -11.0 \\
$\frac{1}{2}^{-}$ & -9.5 & -9.5 & -10.2 & -10.7 \\
$\frac{7}{2}^{-}$ & $-5.3[0.07]$ & -5.3 & $-6.1[0.18]$ & -6.4 \\
$\frac{5}{2}^{-}$ & $-3.4[0.9]$ & -3.4 & $-4.0[1.2]$ & -4.5 \\
$\frac{5}{2}^{-}$ & $-2.3[0.08]$ & -0.3 & $-3.5[0.44]$ & -1.6 \\
$\frac{3}{2}^{-}$ & $-1.2[4.7]$ & -2.2 & - & - \\
$\frac{1}{2}^{-}$ & $-0.9[2.8]$ & -0.9 & & -2.1 \\
$\frac{7}{2}^{-}$ & $-0.4[0.44]$ & -0.4 & $-1.4[?]$ & -1.7 \\
$\frac{3}{2}^{-}$ & - & - & $-0.8[1.8]$ & -3.3 \\
\hline
\end{tabular}

are the known experimental widths obtained from $\mathrm{t}+\alpha\left({ }^{3} \mathrm{He}\right.$ $+\alpha)$ reactions. The nucleon emission thresholds lie higher and so no nucleon widths arise from our calculations. However, those evaluations produced 12 states to $15 \mathrm{MeV}$ excitation in ${ }^{7} \mathrm{Li}$ with the lowest 9 matching known spin-parity states in the spectrum. The next three calculated levels are in the energy region in which two resonant states of undetermined spinparity are known. The five lowest lying states in the known spectrum of ${ }^{7} \mathrm{Be}$ compare reasonably with the MCAS values. The calculations give more states than are known to date above an excitation energy of $\sim 8.5 \mathrm{MeV}$, and there are a few crossings.

\subsubsection{Spectra from ${ }^{3} \mathrm{H}$ - and ${ }^{3} \mathrm{He}-\alpha$ coupling}

To consider other open reaction channels, we have used MCAS to evaluate ${ }^{3} \mathrm{H}$ - and ${ }^{3} \mathrm{He}-\alpha$ scattering using a potential model form of a cluster system. A nuclear interaction was found that gave the results listed in table 2. The calculated results agree well with the known values.

Then we used that interaction with MCAS to find elastic scattering cross sections at three center of mass scattering angles $\left(54.7^{\circ}, 90.0^{\circ}, 125.2^{\circ}\right)$ at which data have been taken. The results of some of those calculations are shown by the solid curves in figure 3 . The comparisons with data are very
Table 2. Spectral properties of ${ }^{7} \mathrm{Li}$ from $\left(\alpha+{ }^{3} \mathrm{H}\right)$ and ${ }^{7} \mathrm{Be}$ from $\left(\alpha+{ }^{3} \mathrm{He}\right)$. Energies are in $\mathrm{MeV}$, widths are in brackets.

\begin{tabular}{lcccc}
\hline \multicolumn{3}{c}{${ }^{7} \mathrm{Li}$} & \multicolumn{3}{c}{${ }^{7} \mathrm{Be}$} \\
\hline$J^{\pi}$ & Exp. & Theory & Exp. & Theory \\
\hline$\frac{3}{2}^{-}$ & spurious & -29.4 & spurious & -28.0 \\
$\frac{1}{2}^{-}$ & spurious & -27.8 & spurious & -26.4 \\
\hline$\frac{3}{2}^{-}$ & -2.47 & -2.47 & -1.59 & -1.53 \\
$\frac{1}{2}^{-}$ & -1.99 & -1.75 & -1.16 & -0.84 \\
$\frac{7}{2}^{-}$ & $2.2(0.06)$ & $2.1(0.08)$ & $3.0(0.18)$ & $3.1(0.18)$ \\
$\frac{5}{2}^{-}$ & $4.1(0.92)$ & $4.2(0.83)$ & $5.1(1.2)$ & $5.1(1.19)$ \\
\hline
\end{tabular}

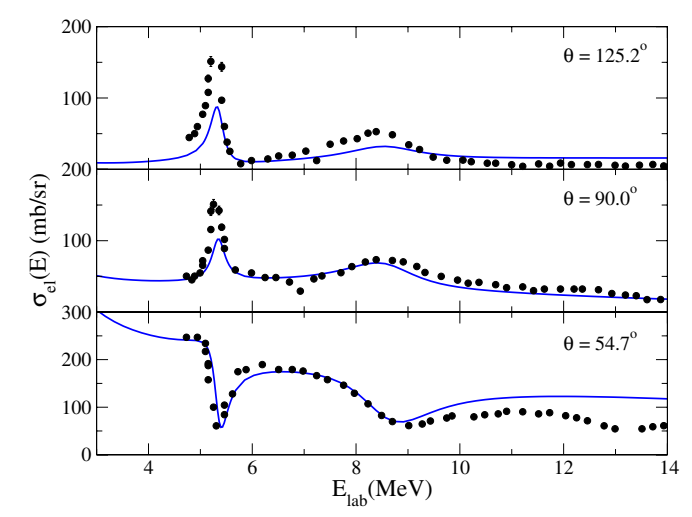

Fig. 3. Cross sections from $\alpha\left({ }^{3} \mathrm{He},{ }^{3} \mathrm{He}\right) \alpha$ scattering.

Table 3. Spectra of ${ }^{7} \mathrm{He}$ and of ${ }^{7} \mathrm{~B}$.

\begin{tabular}{ccccc}
\hline$J^{\pi}$ & ${ }^{7}$ He Exp. & Theory & ${ }^{7}$ B Exp. & Theory \\
\hline$\frac{3}{2}^{-}$ & $0.45(0.15)$ & $0.43(0.1)$ & $2.2(1.4)$ & $2.1(0.19)$ \\
$\frac{7}{2}^{-}$ & - & $1.70(0.03)$ & & $3.0(0.11)$ \\
$\frac{1}{2}^{-}$ & $1.0(0.75)^{a}$ & $2.79(4.1)$ & & $5.4(7.2)$ \\
$\frac{5}{2}^{-}$ & $3.35(1.99)$ & $3.55(0.2)$ & & $5.4(0.34)$ \\
$\frac{3}{2}^{-}$ & $6.24(4.0)^{b}$ & $6.24(1.9)$ & & \\
\hline
\end{tabular}

${ }^{a}$ Observed very recently and interpreted as a $\frac{1}{2}^{-}$state.

${ }^{b}$ Spin-parity of this state is unknown.

good, adding confirmation to our definition of the basic dicluster-interaction potential.

Consider now the results given in table 3 . The spectrum of ${ }^{7} \mathrm{He}$, so far as it is known experimentally, has three resonant states with only the ground being quite narrow. A Paulihindrance effect on the $0 p$ shells again is required to give a ground state that is unbound with respect to neutron emission. With this system, such effects might be a reflection of an exotic and non-compact structure $\left({ }^{6} \mathrm{He}\right)$ being used as a basis in the channel coupling. A similar discussion applies for a proton coupled to ${ }^{6} \mathrm{Be}$ states. However, only the ground state of ${ }^{6} \mathrm{Be}$ is known and it is encouraging that the MCAS calculation has found that resonance energy accurate to $5 \%$. We also predict three more resonances, two of which have widths sufficiently narrow to be detected. 


\subsection{Low energy ${ }^{3} \mathrm{He}-{ }^{4} \mathrm{He}$ radiative capture}

Very recently, Canton, Levchuck, and Shebeko [4] used the MCAS di-cluster model wave function though with an approximate scattering wave function (hence the results are preliminary) to evaluate the radiative capture cross section from which the astrophysical S-factor shown in figure 4 was obtained. Our results overestimate the measured data by only $20 \%$. Given that the bound state of ${ }^{7} \mathrm{Be}$ is not $100 \%$ dicluster, these results are most encouraging. Of note, both scattering and bound state functions can be given by MCAS so that there is no orthogonality problem as in past radiative capture calculations.

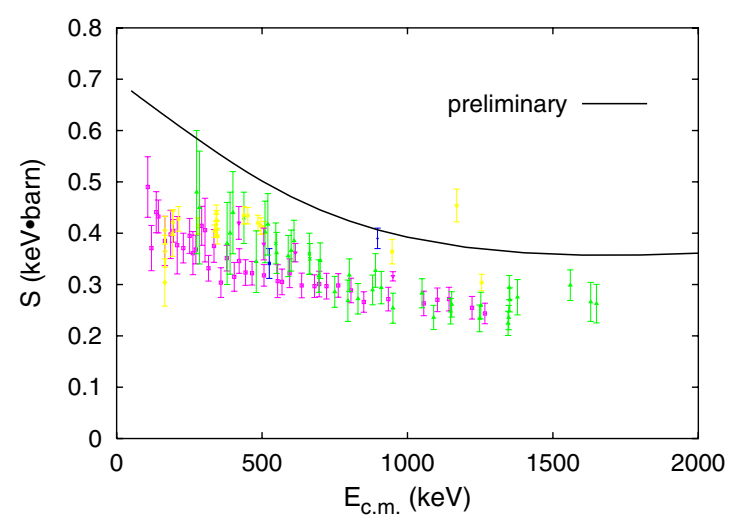

Fig. 4. The astrophysical S-factor from ${ }^{3} \mathrm{He}-{ }^{4} \mathrm{He}$ capture. Data are from figure 3 of ref. [5].

\subsection{Coulomb displacement energies (CDE)}

To minimize effects due to inadequacies in the choice of the nuclear interaction, we consider the CDE of analogues in the two mirror nuclei $\left(X\right.$ and $Y$ ), defined by $\delta_{c}\left(J^{\pi}\right)=$ $E_{J^{\pi}}\left({ }_{N} X_{Z}\right)-E_{J^{\pi}}\left({ }_{Z} Y_{N}\right)$. One may reduce the influence of the chosen nuclear interaction further by considering instead the relative Coulomb shifts for the excited states defined by $\Delta \delta_{c}^{(x)}\left(J^{\pi}\right)=\delta_{c}^{(x)}\left(J^{\pi}\right)-\delta_{c}^{(x)}($ g.s. $)$.

Consider the mass 13 mirrors, ${ }^{13} \mathrm{C}$ and ${ }^{13} \mathrm{~N}$. As in past studies, we fix the matrix of nuclear interaction potentials to be that found for the $n+{ }^{12} \mathrm{C}$ system [1] treated as a three state coupled-channel problem, and from which there were reasonable reproductions of known sub-threshold and resonance states (up to $10 \mathrm{MeV}$ excitation). A uniform charge distribution $\left(R_{c}=2.8 \mathrm{fm}\right)$ was assumed. We have now allowed the nuclear charge distribution to be that of Woods-Saxon (WS) form and have also allowed that distribution to be deformed. The Coulomb radius, diffusivity, and deformation strength were chosen to be $R_{C}=2.8 \mathrm{fm}, a_{C}=0.52 \mathrm{fm}$, and $\beta_{2}^{(C)}=-0.52$.
Table 4. CDE properties from the ${ }^{13} \mathrm{C}$ and ${ }^{13} \mathrm{~N}$ spectra.

\begin{tabular}{cccc}
\hline$J^{\pi}$ & Exp. & $\delta_{c}^{(S q)}\left(J^{\pi}\right)$ & $\delta_{c}^{(D W S)}\left(J^{\pi}\right)$ \\
\hline$\frac{1}{2}^{-}$ & 3.002 & 2.978 & 2.611 \\
$\frac{1}{2}^{+}$ & 2.278 & 2.056 & 1.873 \\
$\frac{5}{2}^{+}$ & 2.697 & 2.500 & 2.303 \\
\hline & Exp. & $\Delta\left(\delta_{c}^{(S q)}\left(J^{\pi}\right)\right)$ & $\Delta\left(\delta_{c}^{(D W S)}\left(J^{\pi}\right)\right)$ \\
\hline$\frac{1}{2}^{+}-\frac{1}{2}^{-}$ & -0.724 & -0.922 & -0.739 \\
$\frac{5}{2}^{+}-\frac{1}{2}^{-}$ & -0.305 & -0.478 & -0.296 \\
\hline
\end{tabular}

In table 4 diverse Coulomb energy properties of three states in the ${ }^{13} \mathrm{C}-{ }^{13} \mathrm{~N}$ mirror nuclei found from the calculated spectra are compared with data. The three most bound states of the mass 13 nuclei are considered. Their spin-parities are listed in column 1 . The data are given in column 2 while the results found using the Coulomb fields of a (undeformed) square well and a deformed WS distribution are listed in columns 3 and 4 respectively. The choice of (square well) Coulomb radius was made to achieve the best spectra and so the CDE for this are quite good. Using the deformed WS charge distribution, CDE remains in reasonable agreement with experiment, though the states are too bound. But the relative Coulomb shifts are in better agreement with observation.

\section{Conclusions}

Applications of the MCAS theory have been made to discern the structure of the compound nuclei underlying various dicluster nuclear systems of light mass. Despite the simplicity of the collective model used to specify the input matrix of interaction potentials, good agreement has been found with available data, even regarding a nucleus lying beyond the proton drip line. Use of MCAS information to evaluate radiative capture cross sections is a promising new development.

\section{References}

1. K. Amos, L. Canton, G. Pisent, J.P. Svenne, D. van der Knijff, Nucl. Phys. A 728, 65 (2003).

2. L. Canton, G. Pisent, K. Amos, S. Karataglidis, J.P. Svenne, D. van der Knijff, Phys. Rev. C 74, 064605 (2006).

3. L. Canton, G. Pisent, J.P. Svenne, K. Amos, S. Karataglidis, Phys. Rev. Lett. 96, 072502 (2006).

4. L. Canton, L. Levchuck, A. Shebeko (private communication).

5. D. Bemmerer et al., Phys. Rev. Lett. 97, 122502 (2006). 